\title{
As dimensões do acesso aos serviços de saúde bucal na mídia impressa
}

\section{Dimensions of oral health services access in printed media}

\author{
Carolina Dutra Degli Esposti \\ Universidade Federal do Espírito Santo. Departamento de Medicina \\ Social. Vitória, ES, Brasil. \\ E-mail: carolinaesposti®gmail.com.br
}

\section{Aline Guio Cavaca}

Universidade Federal do Espírito Santo. Programa de

Pós-graduação em Saúde Coletiva. Vitória, ES, Brasil.

E-mail: alineguicaœhotmail.com

Lezimara Santiago de Andrade Côco

Universidade Federal do Espírito Santo. Vitória, ES, Brasil.

E-mail: lezimarandradeœhotmail.com

\section{Edson Theodoro dos Santos-Neto}

Universidade Federal do Espírito Santo. Programa de

Pós-Graduação em Saúde Coletiva. Vitória, ES, Brasil.

E-mail: edsontheodoro®uol.com.br

\section{Adauto Emmerich Oliveira}

Universidade Federal do Espírito Santo. Programa de Pós-Graduação em Saúde Coletiva. Vitória, ES, Brasil.

E-mail: adautoemmerichœterra.com.br

\section{Correspondência}

Avenida Marechal Campos, 1468, Maruípe. Vitória, ES, Brasil. CEP 29043-900.

\section{Resumo}

As informações divulgadas pela mídia contribuem para construir a representação populacional sobre o Sistema Único de Saúde. Este estudo objetivou analisar a veiculação do tema "acesso aos serviços de saúde bucal" na mídia impressa do Espírito Santo (ES), Brasil. Uma pesquisa de abordagem qualitativa e documental foi realizada, com análise das matérias relacionadas ao acesso aos serviços de saúde bucal veiculadas entre março de 2004 e junho de 2009 nos dois principais jornais do Espírito Santo (A Gazeta e A Tribuna). Para a seleção das matérias utilizamos as palavras-chave: "Dentista”, "Odontologia” e "Saúde Bucal". Foram encontradas 392 notícias, sendo 121 delas sobre o tema “acesso aos serviços de saúde bucal”. Foram selecionadas 41 matérias para esta análise, segundo as regras de exaustividade, representatividade, homogeneidade e pertinência. Aanálise de conteúdo temática dos dados foi realizada seguindo as categorias "disponibilidade", “capacidade de pagar" e "aceitabilidade”. Amaioria das matérias divulgou serviços odontológicos disponibilizados gratuitamente por prefeituras, faculdades, igrejas e ações maçônicas. Percebeu-se maior divulgação sobre políticas públicas de saúde bucal em 2004, que abordavam o acesso ao tratamento odontológico e à água fluoretada. Constatou-se frequente divulgação sobre a disponibilidade dos serviços de saúde bucal, incisiva sinalização dos problemas do setor público e apresentação da saúde suplementar como alternativa para suprir suas ineficiências. Também se verificou poucas matérias que abordaram a dimensão da aceitabilidade. Considera-se que, apesar da considerável disseminação de informações pelos veículos de comunicação no período estudado, torna-se fundamental que a imprensa problematize o acesso aos serviços de saúde bucal em suas dimensões.

Palavras-chave: Acesso aos Serviços de Saúde; Saúde Bucal; Comunicação em Saúde; Meios de Comunicação de Massa. 


\section{Abstract}

The information disseminated by the media contribute to create the population representation about the Health Unified System (SUS). This study aimed to analyze the publication of the theme "access to oral health services" in the print media of the Espírito Santo (ES), Brazil. We used a qualitative and documental approach to analyze the news on the access to oral health services disseminated between March 2004 and June 2009 in the two main newspapers of Espírito Santo, Brazil (A Gazeta and A Tribuna). To select the materials we used the keywords "Dentist", "Dentistry" and "Oral Health". Of the 392 news found, 121 were about "access to oral health services". We selected 41 pieces for this analysis according to the rules of completeness, representativeness, consistency and relevance. The thematic content analysis of the data was performed according to the following categories: "availability", "acceptability" and "affordability". Most part of the news published was about dental services provided for free by municipalities, colleges, churches and Masonic actions. We observed more attention given to public dental health policies in 2004, addressing access to dental treatment and to fluoridated water, and also noticed frequent disclosure about the availability of oral health services, emphasizing thus the problems of public sector and supplementary health insurance as an alternative to address its inefficiencies. Few pieces addressed acceptability. We considered that, despite the considerable dissemination of information by the media on the period of our study, it is essential that the press problematizes access to oral health services in its dimensions.

Keywords: Health Services Accessibility; Oral Health; Health Communication; Mass Communication.

\section{Introdução}

O papel informativo e educativo da mídia favorece a discussão sobre o acesso aos serviços de saúde bucal, pois além de exercer o poder de pautar ao público os assuntos de interesse (agenda-setting), a mídia direciona quais partes da realidade se tornarão visíveis (enquadramento), possuindo um lugar de suma importância na construção da representação social e na divulgação do acesso aos serviços de saúde para a sociedade. A esse respeito, sabe-se que quanto menor é a experiência direta do indivíduo em relação a um determinado assunto, maior é sua dependência dos meios de comunicação para obter informações e interpretações referentes a esse tema (Wolf, 1999).

Entende-se que, para a efetivação dos princípios ético-políticos do Sistema Único de Saúde (SUS), há necessariamente o envolvimento de processos comunicacionais relacionados tanto a sua visibilidade pública quanto à divulgação de informações e conhecimentos que permitam à população configurar seu entendimento da saúde pública. Todavia, percebe-se que as principais imagens e informações divulgadas pela mídia sobre o SUS geralmente estão associadas às mazelas e dificuldades do setor, contribuindo para a construção de uma representação pouco crítica sobre esse campo (Oliveira, 200o).

O estudo e a avaliação do acesso aos serviços de saúde e a definição de uma agenda para as políticas desse setor, baseada na equidade, dependem da clareza dos conceitos e dos modelos explicativos a serem empregados (Travassos; Castro, 2012; Thiede; Akewengo; McIntyre, 2014). A partir de Travassos e Castro (2012), é possível compreender que o conceito de acesso é complexo e varia, segundo diversos autores, ao longo do tempo e de acordo com o contexto. Por vezes, acaba sendo empregado de forma imprecisa e com pouca clareza em sua relação com a utilização de serviços de saúde, dificultando o emprego de metodologias adequadas à sua análise e avaliação.

Segundo Thiede, Akewengo e McIntyre (2014), a equidade no cuidado à saúde é frequentemente definida com base no acesso aos serviços de saúde. Os autores reconhecem o caráter multifatorial do 
conceito de acesso e buscam indicar como operacionalizar a superação das iniquidades do acesso presente nos sistemas de saúde, a partir de uma discussão que inclui a relação entre os indivíduos e o sistema. 0 acesso não é sinônimo de utilização de serviços, pois sofre influência de diversos fatores que modificam a oportunidade de utilização das pessoas - por exemplo, a liberdade em fazê-lo, fator que pode ampliar ou diminuir a equidade. Segundo esses autores, os determinantes do acesso podem ser resumidos em três dimensões: "disponibilidade", "capacidade de pagar" e "aceitabilidade".

A disponibilidade pode ser entendida como existência de um serviço específico de saúde ao alcance do potencial usuário, no momento certo e em local adequado, e que inclui aspectos como sistemas de marcação e horário de funcionamento, em relação à disponibilidade de cuidado no momento adequado; e natureza e extensão da oferta de serviços em comparação às necessidades de saúde da população a qual se destinam (Thiede; Akewengo; McIntyre, 2014).

A capacidade de pagar reflete o grau de financiamento público do sistema e é definida como a capacidade do usuário incorrer com os custos diretos e/ou indiretos da utilização dos serviços, sendo capaz de mobilizar recursos financeiros, caso requeridos, para atender às suas necessidades em saúde, como gastos com honorários profissionais, exames diagnósticos, medicamentos e a perda de produtividade em decorrência do tempo despendido com viagens e espera por atendimento (Thiede; Akewengo; McIntyre, 2014).

Já a aceitabilidade relaciona-se a fatores subjetivos, culturais e sociais que influenciam a avaliação dos indivíduos sobre os serviços de saúde, as expectativas dos pacientes sobre a organização do serviço, a aceitação quanto ao cuidado prestado e ao respeito no atendimento, assim como a escuta de suas queixas (Thiede; Akewengo; McIntyre, 2014).

Deve-se reconhecer que a partir dessas dimensões configuram-se as barreiras de acesso geográficas, financeiras, organizacionais e de informação, com reflexos práticos para a equidade no acesso aos serviços de saúde (Travassos; Castro, 2012). Dessa maneira, a informação é um importante determinante da qualidade do sistema de saúde (Thiede;
Mcintyre, 2008), considerada elemento central que perpassa as dimensões do acesso. A informação sobre o processo saúde-doença e sobre as opções de cuidado disponíveis capacita as pessoas para escolhas no contexto dos cuidados de saúde, afetando o acesso de forma expressiva.

No âmbito da atenção à saúde bucal no Brasil, a assistência odontológica é repleta de barreiras que dificultam o seu acesso. Em relação à organização das políticas de saúde bucal no país, durante muitos anos a inserção das práticas odontológicas no SUS deu-se de maneira paralela e afastada do processo de organização dos demais serviços de saúde (Brasil, 2006). Contudo, a Política Nacional de Saúde Bucal - Programa Brasil Sorridente apontou para a reorganização da atenção em todos os níveis, incluindo a ampliação da Atenção Básica, por meio da Estratégia Saúde da Família (ESF), a ampliação e a qualificação da Atenção Especializada, através da implantação de Centros de Especialidades Odontológicas (CEOs) e dos Laboratórios Regionais de Próteses Dentárias, e a viabilização da fluoretação das águas nas estações de tratamento de abastecimento público (Brasil, 2004).

Diante dessas considerações, o objetivo deste estudo foi analisar a veiculação do tema "acesso aos serviços de saúde bucal” na mídia impressa do Espírito Santo, Brasil, a partir do lançamento do Programa Brasil Sorridente em 2004 e nos cinco anos subsequentes à sua implantação, a fim de discutir as dimensões do acesso mais visibilizadas.

\section{Metodologia}

Foi realizada uma pesquisa documental, com abordagem qualitativa, a partir da análise temática de conteúdo (Bardin, 2009) das matérias relacionadas ao acesso aos serviços de saúde bucal veiculadas no período de março de 2004 a junho de 2009, nos jornais A Gazeta e A Tribuna, os principais do Espírito Santo. Esses anos são subsequentes para a implantação do Brasil Sorridente (Brasil, 2004).

Para coleta de dados do jornal A Tribuna, foi utilizado o banco de dados digital fornecido pelo jornal, e a seleção das notícias foi realizada por meio de um Programa de Busca Inteligente, desenvolvido 
pelo Laboratório de Recuperação Inteligente da Informação da Universidade Federal do Espírito Santo (UFES), o qual utiliza a busca de informações baseada não apenas em palavras-chave, mas considerando a semântica subjacente à consulta feita pelo usuário, resgatando a informação por aproximações sucessivas, o que facilita o processo de busca (Azevedo et al., 2005). Já as matérias do jornal A Gazeta foram selecionadas pelo programa de busca do próprio jornal. Assim, inicialmente fez-se um levantamento retrospectivo das matérias dos dois periódicos que apresentavam em seu conteúdo as palavras-chave: "Dentista”, "Odontologia” e "Saúde Bucal". A partir disso, selecionaram-se as notícias que abordavam o acesso aos serviços de saúde bucal.

A coleta de dados inicial totalizou 392 matérias publicadas sobre saúde bucal no período analisado, sendo 178 matérias no jornal A Gazeta e 214 matérias no jornal $A$ Tribuna. A partir desse conjunto, foram selecionadas aquelas que tratavam do acesso aos serviços de saúde bucal, totalizando 121 matérias.

Assim organizado, o material empírico foi submetido à análise temática de conteúdo, que representa a análise dos temas ou itens de significação numa unidade de codificação previamente determinada, visando "descobrir os 'núcleos de sentido’ que compõem a comunicação e cuja presença ou frequência de aparição, podem significar alguma coisa para o objetivo analítico escolhido" (Bardin, 2009, p. 131, grifo da autora). Possui, dessa forma, capacidade de produzir inferências de um texto focal para seu contexto social de maneira objetivada, além de poder ser utilizada para detectar tendências e modelos na análise de critérios de noticiabilidade, enquadramentos e agendamentos na pesquisa jornalística, estabelecendo alguns parâmetros culturais implícitos e a lógica organizacional por trás das mensagens (Minayo, 2008; Herscovitz, 2007; Shoemaker; Reese, 1996).

Como preconizado por Bardin (2009), a análise de conteúdo foi iniciada a partir da pré-análise, a qual corresponde à fase de organização, permitindo considerar as intuições e sistematizar as ideias iniciais. A leitura flutuante dos 121 documentos por três pesquisadoras treinadas garantiu o contato com o material e gerou impressões e orientações que permitiram selecionar o corpus de 41 matérias para serem submetidas aos procedimentos analíticos, atendendo às regras de exaustividade, representatividade, homogeneidade e pertinência defendidas por Bardin (2009).

A segunda fase, de exploração do material, consistiu na aplicação sistemática das decisões tomadas na pré-análise, relacionadas à codificação e à categorização do material. O tema foi definido como unidade de registro, que foi identificado no corpus, permitindo o recorte dos trechos dos documentos e sua transcrição em uma grade de análise, classificados a posteriori em categorias definidas a partir das peculiaridades das matérias.

Na terceira e última fase, relativa ao tratamento dos resultados, inferência e interpretação, foi possível interpretar as mensagens latentes nas matérias e fazer inferências a partir da teoria sobre o acesso proposta por Thiede, Akewengo e McIntyre (2014) e dos conceitos elaborados por Travassos e Castro (2012), permitindo a construção da análise a partir de três categorias: "disponibilidade", "capacidade de pagar" e "aceitabilidade", destacando os alcances e limites apresentados nos trechos. Todas as matérias foram codificadas seguindo a inicial do nome do jornal ( $\mathrm{T}=$ A Tribuna; $\mathrm{G}=$ A Gazeta), e a identificação do interlocutor da fala.

A pesquisa foi aprovada pelo Comitê de Ética em Pesquisa do Centro de Ciências da Saúde da UFES, sob o número de registro $\mathrm{n}^{0} 175$ /o9, e foi concedida autorização formal dos jornais selecionados para a sua realização.

\section{Resultados e discussão}

As 121 matérias relacionadas ao tema "acesso aos serviços de saúde bucal” tratavam de notícias, reportagens e, principalmente, notas. Em grande parte, divulgavam os serviços de Odontologia disponibilizados gratuitamente em vários cenários: prefeituras, faculdades, igrejas e ações maçônicas. Constatou-se um grande número de matérias no ano de 2004 sobre o Programa Brasil Sorridente, 
abordando a oferta e o acesso ao tratamento odontológico e à água fluoretada, na ocasião do lançamento da política.
As dimensões do acesso foram apresentadas a partir da perspectiva de seus alcances (destacando potencialidades e práticas efetivas) e/ou de seus

Figura I. Matriz de Análise

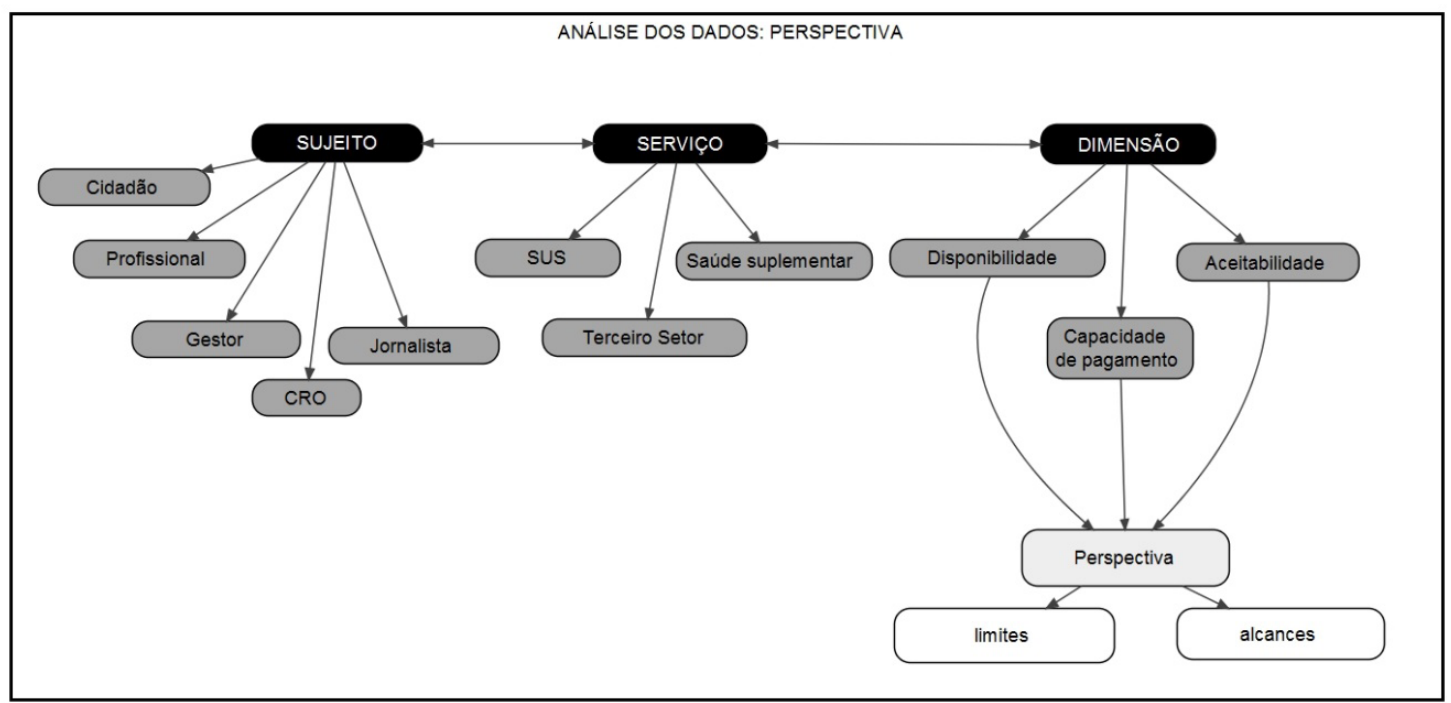

limites (representações das barreiras de acesso). Dessa maneira, identificaram-se nos resultados a categorização da dimensão, a perspectiva, o lugar de interlocução do sujeito da fala e a que tipo de serviço se referia (Figura 1).

\section{Disponibilidade}

O conceito de acesso aos serviços de saúde engloba, de forma ampla, a relação geográfica entre as instituições físicas de saúde e os indivíduos que dela necessitam, como distância e opções de transporte (Thiede; Akewengo; McIntyre, 2014).

Quanto à disponibilidade, a maioria das notícias tratava da oferta de serviços públicos, informando à população sobre o atendimento básico e especializado em saúde bucal disponível no SUS, assim como a distribuição gratuita de produtos de higiene bucal à população, como se percebe nos trechos a seguir:

[...] Para cuidar bem do corpo e da aparência, quase todas as prefeituras municipais oferecem serviços básicos, como aplicação de flúor, obturações e limpeza para retirada de dor, gratuita- mente. Basta ir até uma unidade de saúde mais próxima [...] (G33, jornalista).

Com o CEO, a população terá um tratamento dentário diferenciado, no mesmo nível encontrado nas clínicas particulares [...] ( $\mathrm{T}_{79}$, coordenador de CEO).

A disponibilidade constitui-se na representação da existência ou não do serviço de saúde no local apropriado e no momento em que é necessário (Thiede; Akewengo; McIntyre, 2014). De acordo com Peres et al. (2012), é importante analisar o acesso da população brasileira aos serviços odontológicos, pois os problemas de saúde bucal relacionam-se diretamente à qualidade de vida dos indivíduos e existem desigualdades sociais na sua utilização. No Brasil, observa-se o aumento da oferta de serviços odontológicos, a partir da implantação do Brasil Sorridente, com a implementação dos CEOs e a ampliação do número de Equipes de Saúde Bucal (ESB) na ESF, visando garantir a integralidade dos cuidados em saúde bucal. Apesar da criação e ampliação dos serviços, 
ainda são necessários mais esforços para reduzir as desigualdades sociais no acesso e no processo do cuidado (Rocha; Goes, 2008).

Ao refletir sobre o papel das ESBs na ampliação do acesso aos serviços de saúde bucal, principalmente a partir do lançamento da Política Nacional de Saúde Bucal, deve-se levar em conta que as matérias destacaram que muitas pessoas ainda não têm acesso ao cuidado da saúde bucal. Pode-se transcrever um trecho que menciona esse fato:

Cerca de 503 mil capixabas nunca foram ao consultório do dentista, o que corresponde a 15,23\% da população do Espírito Santo [...] Embora seja menor do que o registrado em 1998 (17,44\%) ainda preocupa o Conselho Regional de Odontologia (CRO-ES) (G44, jornalista).

Tal como informado pela mídia, existe a discussão sobre o fato de que a oferta de serviços odontológicos é insuficiente para garantir o acesso a esses serviços, visto que uma parcela expressiva da população brasileira ainda não tem acesso aos serviços odontológicos. Sobre esse fato, a Pesquisa Nacional por Amostra de Domicílios (PNAD) dos anos de 2003 e 2008 mostrou um aumento da utilização dos serviços odontológicos em todas as idades, no período entre os anos de 1998 e 2008 , o que pode ser atribuído também ao aumento na oferta de serviços públicos de saúde bucal. A análise por grupo etário revelou, no entanto, que a proporção de indivíduos que nunca foi ao dentista foi expressivamente maior até os seis anos de idade, quando comparada aos outros grupos, tanto em 2003 (71,5\%) quanto em 2008 (66,8\%) (Peres et al., 2012).

Outro aspecto descrito acerca da disponibilidade relaciona-se à organização dos serviços. Nota-se a divulgação, pela mídia, da ampliação dos horários de atendimento, e a garantia de oferta mesmo em localidades distantes dos centros urbanos, como segue a seguir:

[o atendimento] passará a ser feito também aos sábados e domingos. A medida é para beneficiar também pessoas que moram na área rural $e$ aqueles que trabalham durante a semana e por isso não conseguem marcar consultas ( $\mathrm{G}_{105}$, jornalista).

Queremos ainda neste ano passar a atender à noite também (G105, Coordenadora Municipal de Saúde Bucal).

Dentistas sobre rodas na zona rural. A iniciativa visa a garantir o controle das cáries em crianças e adultos na população que tem dificuldade de locomoção até os postos de saúde da sede do município (T85, jornalista).

A análise das notícias permite a reflexão sobre a possibilidade de garantia do acesso universal, instituindo ferramentas para a superação das barreiras geográficas e organizacionais, como pretende a política. Essa inferência é possível, pois a disponibilidade compreende também a relação entre tipo, abrangência, qualidade e quantidade dos serviços de saúde prestados, assim como o nível pelo qual os serviços de saúde se ajustam às necessidades dos indivíduos (Thiede; Akewengo; McIntyre, 2014) - por exemplo, o horário de funcionamento (especialmente para quem trabalha integralmente em período diurno). Quanto à organização, variáveis mensuráveis que afetam a entrada no sistema de saúde podem incluir tempo de viagem, espera para o agendamento de consultas e para o atendimento.

O levantamento epidemiológico nacional de saúde bucal, realizado no Brasil em 2003, e os dados do suplemento saúde da PNAD de 1998 apontaram que os principais problemas de saúde bucal eram a cárie dentária e suas consequências, além da falta de acesso às ações e aos serviços de saúde bucal. Nesse período entre 1998 e 2003, houve redução da população de brasileiros que nunca foram ao dentista em $15 \%$, demonstrando que o acesso aos serviços de saúde bucal aumentou. Contudo, os dados da PNAD de 2008 destacaram que os problemas odontológicos são a terceira razão para a procura de serviços de saúde, com baixo acesso aos serviços mesmo para aqueles que buscaram atendimento odontológico (40,0\%) (Peres et al., 2012). Já o último levantamento epidemiológico nacional, realizado em 2010, mostrou importante tendência de declínio da cárie dentária 
e melhoria do acesso aos serviços de saúde bucal aos 12 anos de idade e para a faixa etária de 15 a 19 anos, tendo sido ainda mais acentuado em adultos (35 a 44 anos). No entanto, foram reveladas marcantes diferenças regionais na prevalência e gravidade da cárie, indicando como necessárias políticas públicas voltadas para a redução das desigualdades na saúde e para o acesso aos serviços de saúde (Roncalli, 2011).

Diante do fato de que a prevalência de doenças bucais ainda é alta no Brasil e de que nem todos conseguem utilizar os serviços de saúde bucal no momento ideal, apesar do aumento na oferta de serviços com a implementação do Brasil Sorridente, as matérias ofereciam como opção os serviços ofertados pela saúde suplementar, por ações filantrópicas e também por instituições de ensino superior. Dessa forma, a população que se via excluída da organização dos serviços públicos de saúde bucal contaria com outras opções de serviços, as quais foram frequentemente divulgadas pela mídia impressa, como se pode destacar:

Tratamento dentário mais fácil. Planos odontológicos estão ampliando serviços e cobrando mensalidades a partir de RS 18,oo. Cada vez mais presente no mercado para suprir uma carência de grande parte da população, os planos odontológicos estão se consolidando como uma opção de custo reduzido para quem quer fazer restaurações, cirurgias e até aparelho nos dentes (T72, jornalista).

Uma alternativa pode ser buscar um tratamento nas faculdades que, num misto de trabalho social e prática profissional para seus alunos, oferecem vários serviços à comunidade (T32, jornalista).

Cortes de cabelo, exames oftalmológicos, atendimento odontológico e emissão de documentos. Tudo isso será oferecido gratuitamente na II ação maçônica (T35, jornalista).

Esses serviços apresentam-se como possibilidade de superação das barreiras de acesso, porém evidenciam a falta de cumprimento de princípios do SUS, como a integralidade e a universalidade, sendo uma opção para aqueles mais favorecidos economicamente. De acordo com a Agência Nacional de Saúde Suplementar (ANS), entre março de 2004 e março de 2014, o número de beneficiários em planos privados exclusivamente odontológicos cresceu aproximadamente 10 vezes no ES, sendo mais que o dobro do crescimento observado para o Brasil no mesmo período (ANS, 2014). Assim como discutido pela mídia, pode-se relacionar esse crescimento às dificuldades ainda existentes de acesso aos serviços odontológicos no sistema público.

De acordo com Santos (2012), o jornal impresso tem grande responsabilidade em oferecer à população uma informação com maior importância discursiva, permitindo ao público a interpretação dos efeitos dos serviços disponíveis e como acessá-los. Entende-se o acesso à saúde bucal no sistema público como a oportunidade de utilização dos serviços em circunstâncias que permitam sua utilização apropriada, uma vez que o SUS, em suas atribuições, é direito de todos. Sendo a informação essencial para a compreensão de cada uma das dimensões do acesso, a mídia exerce um papel importante na promoção dessas informações, mesmo que em determinados momentos chegue de forma negativa aos cidadãos, principalmente ao referir-se à saúde pública (Cavaca et al., 2012).

Oliveira (2013) mostra a disparidade entre as notícias sobre saúde, do ponto de vista da mídia e dos mediadores da saúde, ou seja, o campo concentra-se em informar aos indivíduos suas particularidades, inovações e vantagens, ao passo que a mídia utiliza os mesmos atributos frequentemente com interesses diversos, passando a imagem de um sistema que não funciona adequadamente. Portanto, nota-se nas matérias analisadas a preocupação em divulgar os desvios do SUS para uma população que não tem acesso à informação clara dos serviços oferecidos.

\section{Capacidade de pagar}

O SUS, em suas atribuições, deveria garantir o acesso universal e um cuidado integral e de qualidade a todos os brasileiros. No entanto, verificou-se nos textos analisados que muitos indivíduos com necessidade de tratamento odontológico utilizaram 
serviços privados, mesmo com dificuldades financeiras para custeá-lo. Tais situações se relacionam às capacidades restritas e individuais do financiamento dos serviços de saúde por desembolso direto de parte da população brasileira para comprar os produtos básicos de higiene bucal e também para utilizar os serviços ofertados pelo setor público. Houve relatos de que o atendimento foi realizado desconsiderando os dispositivos de prevenção primária, que foram substituídos por tratamentos mais invasivos, dispendiosos, mutilantes e dolorosos, como se pode notar nos trechos a seguir:

Ministério da Saúde revela que 40\% da população brasileira não têm acesso à escova e à pasta de dente. [...] isso ocorre não apenas por falta de orientação, mas porque as pessoas não têm condições financeiras para comprar os produtos básicos para higiene bucal (G19, jornalista).

Eles só faziam limpeza e aplicavam a medicação para dor, mas não resolviam o problema. Tive que pagar para arrancar meu dente (G155, usuária).

Nunca tive condições de pagar dentista particular. Quando cheguei ao posto disseram que o jeito era arrancar meu dente (G155, usuário).

O acesso à saúde envolve múltiplos aspectos, de ordem socioeconômica e cultural, que extrapolam a assistência à saúde. Acesso implica garantia de ingresso do indivíduo no sistema de saúde, ou a utilização de bens e serviços considerados socialmente importantes, sem obstáculos físicos, financeiros ou de outra natureza (Matos et al, 2002). A capacidade de pagar compreende a relação entre o custo de utilização dos serviços de saúde e a capacidade dos indivíduos em pagar. São consideradas nessa dimensão as despesas com consultas, gastos com medicamentos, exames complementares, com alimentação especial, transporte e até mesmo perda de produtividade e de renda salarial devido ao tempo de espera por atendimento (Thiede; Akewengo; McIntyre, 2014).

Um tema importante destacado pelos periódicos analisados é a influência de fatores sociais no acesso à saúde bucal: quanto maior a renda e a escola- ridade, melhor o acesso ou utilização dos serviços. Isso destaca a limitação do SUS em garantir o acesso universal aos brasileiros e demonstra claramente a iniquidade no acesso aos serviços odontológicos, como informado no trecho destacado:

O acesso ao tratamento odontológico éproporcional à renda. Boa parte dos capixabas que nunca foram ao dentista, 28,9\%, vivia com até um salário mínimo na época da pesquisa (2003) (G44, jornalista).

Se por um lado quem depende dos serviços públicos tem dificuldade até para manter os dentes na boca, quem pode pagar por um dentista especializado tem o céu como limite [...] (G156, jornalista).

Essa é uma realidade que preocupa os pesquisadores de todo o mundo, pois a existência de desigualdades no acesso aos serviços de saúde bucal parece ser mais comum do que se imagina. O estudo deHosseinpoor, Itani e Petersen (2012), desenvolvido a partir dos resultados da Pesquisa Mundial de Saúde de 2002/2004, mostrou desigualdades na cobertura de serviços de saúde bucal entre e dentro dos países: nações mais ricas tiveram maior cobertura de serviços de saúde bucal para aqueles com necessidade percebida do que os países mais pobres. Também existem desigualdades socioeconômicas em favor dos mais ricos dentro da maioria dos países, com maiores níveis de desigualdade relativa em países de baixa renda do que nos de maior renda; e, no geral, menor cobertura e maior desigualdade relativa foram observadas em países de baixa renda. No Brasil, entre 2008 e 2009, o tratamento odontológico buscado no serviço particular representou $4 \%$ do gasto familiar com saúde e $7 \%$ da renda familiar mensal dos brasileiros (IBGE, 2012).

Durante a análise, observou-se o foco no prenúncio de que o tratamento odontológico predominantemente privado apresenta restrição para a população com poder aquisitivo mais baixo, como se pode notar no trecho a seguir:

Dentista grátis para crianças [...] Essa iniciativa vai ajudarmuito nossas crianças e adolescentes. Sabe- 
mos que eles não têm condições de pagar uma consulta e realmente necessitam de assistência (G146, Diretor da escola atendida pelo projeto social).

No trecho, verifica-se um enfoque crítico da mídia que chega à população ao tentar abranger os limites e os alcances para o acesso aos serviços de saúde bucal. Apesar de Peres et al. (2012) terem observado a redução nas desigualdades no acesso e na utilização dos serviços odontológicos entre os indivíduos de maior e menor renda no Brasil entre 2003 e 2008 , em determinados momentos a mídia apresenta o SUS não como um bem público, mas como um serviço gratuito. Oliveira (2000) corrobora essa ideia, afirmando que as imagens geradas pela mídia colocam o serviço público de saúde de forma negativa, citando-o como ineficiente, com mau atendimento, enquanto retrata o privado como modelo de organização e conforto, defendendo interesses desse setor e dando enfoque também às ações de instituições não governamentais. Como podem ser notadas, as notícias sobre serviços filantrópicos foram bem elucidativas nos dois jornais analisados, com destaque para as ações sociais de grupos de profissionais, igrejas e faculdades como forma de promover a saúde bucal aos menos favorecidos.

Foram encontrados alguns informes publicitários de prefeituras divulgando seus serviços e inovações em saúde bucal, nos quais pode ser visto, além do marketing, uma disponibilização de informações sobre serviços prestados e como consegui-los naqueles municípios. Porém, a maneira de divulgação do acesso aos serviços odontológicos, na maioria das vezes, adota a conotação de marketing social e não os contextualiza como um direito do cidadão, tal como preconizado pelo Brasil Sorridente. Tal perspectiva tem se tornado mais frequente também em práticas comunicativas institucionais, as quais delegam a profissionais especializados em marketing funções de comunicadores. Assim sendo, substituem-se processos e práticas de interlocução por venda de ideias de convencimento de adoção de determinados comportamentos "ideais" e de consumo de determinados produtos (Araújo; Cardoso, 2007).

Uma comunicação em saúde crítica e emancipadora precisa considerar os contextos sociais dos sujeitos, levar em consideração os processos políticos de estabelecimento de prioridades, suas condições subjetivas de produção de sentidos e as relações de poder que permeiam as práticas comunicativas (Araújo; Cardoso, 2007). A divulgação midiática de serviços de saúde bucal, da mesma maneira, não pode apresentá-los como um benefício ou favor prestado à população por parte dos governos. 0 acesso a esses serviços trata-se de direito de todos, e a mídia tem o papel de veiculá-los de maneira crítica, não se prestando como veículo de propaganda política em hipótese alguma.

\section{Aceitabilidade}

A categoria "aceitabilidade" foi citada poucas vezes nos documentos analisados (em apenas três das 41 matérias analisadas). No conjunto da análise das categorias "disponibilidade" e "aceitabilidade", a mídia informou que a oferta de serviços de saúde bucal no SUS nem sempre atende às necessidades da população, caracterizando-os como precários. Essa insatisfação com o serviço recebido foi destacada também pelos usuários, que nem sempre se mostravam satisfeitos com os procedimentos disponibilizados na rede pública de saúde, como pode ser captado do seguinte trecho:

Tem que participar de palestra, mas as pessoas trabalham. Além disso é um trabalho danado e sófazem limpeza. Quem já está com problema no dente tem que arrancar (G155, usuário).

A relação entre as atitudes dos profissionais de saúde e dos indivíduos, influenciada por fatores como idade, sexo, etnia, idioma, crenças culturais, condição socioeconômica etc. definem a aceitabilidade. Um dos pontos principais no conceito de aceitabilidade é o respeito mútuo entre profissionais de saúde e usuários dos serviços. De acordo com Thiede, Akewengo e McIntyre (2014), a aceitabilidade compreende a natureza dos serviços prestados e o modo como são percebidos pelos indivíduos e comunidades. Além disso, o Brasil Sorridente, como modelo de reorientação das práticas em saúde bucal no SUS (Brasil, 2004), visa ampliar a atenção à saúde bucal e o enfrentamento contra a cultura 
do odontocentrismo e da extração-mutilação dentária que permeia o imaginário odontológico, tanto subjetivo quanto midiático. Trata-se de desafiadora proposta, uma vez que as teorias odontológicas hegemônicas reproduzem esse centramento dentário (Botazzo, 2006).

No imaginário odontológico da população, a extração dental é um mediador para a retirada da dor, resolvendo o problema a um custo operacional muito baixo. Historicamente, esse tipo de procedimento é, geralmente, buscado pelo cidadão no serviço público como válvula de escape para o caso de dor (Emmerich, 200o). Ainda segundo o autor, cuidar da saúde bucal é uma atividade necessária a todos os indivíduos, independente de sua faixa etária, classe social ou nível de escolaridade. Contudo, uma parcela significativa da população brasileira, principalmente de nível socioeconômico mais baixo, enfrenta dificuldades de acesso a serviços especializados de odontologia, quer seja pela carência de informação sobre educação em saúde, quer pela falta de oferta ou barreiras de acesso aos serviços públicos de saúde bucal.

Outro fator parece interferir no comportamento de grande parte dos indivíduos que buscam atendimento odontológico: eles acreditam que serão submetidos a algum tipo de desconforto durante o tratamento. $\mathrm{O}$ medo pode estar relacionado ao ambiente, aos aparelhos, à possibilidade de já terem tido experiências negativas e também pelo fato de muitos procurarem o profissional quando a dor já está instalada (Emmerich, 200o). Além disso, há frequente veiculação midiática da figura do dentista associada ao medo e à dor (Henriquez, 1993), o que contribui para a perpetuação de tal imaginário.

Outra matéria destaca a importância do acesso aos serviços de saúde bucal de qualidade do ponto de vista dos usuários. É gerada uma satisfação nas pessoas que conseguem utilizar o serviço e um medo da possibilidade de não recebê-lo, como no trecho a seguir:

No programa de saúde bucal aqui do município, eu posso levar meus meninos e eles são bem atendidos. Não gostaria que os serviços fossem suspensos (G165, usuária).
A mídia tem o intuito de levar informações aos usuários dos serviços de saúde e facilitar a interpretação delas de acordo com o que veicula. Os jornais analisados cobriram superficialmente situações nas quais os usuários do sistema público fazem boa referência aos serviços que utilizam, uma vez que notícias com caráter negativo geram mais discussão e prendem mais a atenção dos leitores, conforme preconiza uma conhecida expressão do campo jornalístico: "notícia ruim é notícia boa".

A mídia é uma forma dos usuários identificarem suas localizações e opiniões sociais. Além de constituir fonte de formação de crenças e atitudes, pode representar o pensamento coletivo da sociedade (Ronzani et al., 2009). Portanto, notícias com caráter demonstrativo das ações positivas do SUS e do Brasil Sorridente devem ser exploradas, para que os usuários tenham ciência não apenas da disponibilidade dos serviços, mas principalmente da importância e da qualidade deles.

\section{Considerações finais}

A complexidade do conceito de acesso pode dificultar a sua adequada aplicação pela mídia impressa brasileira, levando-a a uma interpretação estereotipada sobre o tema. Deve-se considerar também como um problema a ser enfrentado no âmbito das políticas de saúde no SUS o fato da profissão odontológica ter se constituído desconectada da realidade epidemiológica e social do país.

0 Brasil Sorridente, nesse sentido, visa à mudança no modo de desenvolvimento de ações de saúde bucal, passando a ser pautada pelos princípios da gestão participativa, da ética, do acesso, do acolhimento, do vínculo e da responsabilidade profissional. Essa política busca oferecer o acesso universal à assistência a partir de ações individuais e coletivas, atendendo à demanda expressa ou reprimida e responsabilizando-se por todos os problemas de saúde da população de um determinado espaço geográfico. Nesse contexto, entende-se que a mídia representaria também uma parceira importante nas ações intersetoriais de promoção da saúde e na divulgação das políticas e atividades assistenciais de saúde.

Os resultados encontrados, referentes às pu- 
blicações nos primeiros anos após o lançamento do Brasil Sorridente, demonstraram que a mídia é essencial para o conhecimento da população a respeito da oferta dos serviços de saúde bucal na rede pública. Porém, ainda é necessária uma abordagem mais positiva a respeito do SUS que leve a população a ter interesse na procura desses serviços.

Sendo assim, torna-se imprescindível que se questione e se reflita a respeito do entendimento sobre os serviços considerados gratuitos pelo SUS, constantemente veiculados pela mídia, conforme verificado neste estudo, a despeito da garantia do acesso aos serviços de saúde ser um direito social. O SUS é um sistema público de saúde, financiado pelos orçamentos da União, dos Estados e dos Municípios, oriundos da arrecadação de impostos no país. Trata-se, portanto, de um direito de cidadania o acesso à assistência a saúde bucal de qualidade, equânime e integral, sendo essa representação do gratuito extremamente enviesada, o que contribui para a manutenção do imaginário popular de um SUS de má qualidade, voltado somente para a população mais pobre.

\section{Referências}

\section{ANS - AGÊNCIA NACIONAL DE SAÚDE}

SUPLEMENTAR. Dados e indicadores do setor:

beneficiários de planos privados de saúde. Rio de Janeiro: ANS, 2014. Disponível em: <http://www. ans.gov.br//materiais-para-pesquisas/perfil-dosetor/dados-e-indicadores-do-setor\#>. Acesso em: 6 ago. 2014 .

ARAÚJO, I. S.; CARDOSO, J. M. Comunicação e saúde. Rio de Janeiro: Fiocruz, 2007.

AZEVEDO, L. et al. Recuperação de informação através do processo de aproximações sucessivas. In: XXI CONGRESSO BRASILEIRO DE BIBLIOTECONOMIA, DOCUMENTAÇÃO E CIÊNCIA DA INFORMAÇÃO, 21., 2005, Curitiba. Anais... Curitiba: CBBD, 2005. Disponível em: <http://www.multicast.com.br/sergio/ri-artigoaproximacoes-sucessivas-cbbd2005.pdf $>$. Acesso em: 25 abr. 2014.

BARDIN, L. Análise de conteúdo. Lisboa: Edições 70, 2009 .
BOTAZZO, C. Sobre a bucalidade: notas para a pesquisa e contribuição ao debate. Ciência \& Saúde Coletiva, Rio de Janeiro, v. 11, n. 1, p. 7-17, 2006.

BRASIL. Ministério da Saúde. Diretrizes da Política Nacional de Saúde Bucal. Brasília, DF, 2004. Disponível em: <http://conselho.saude.gov. br/web_comissoes/cisb/doc/politica_nacional. pdf $>$. Acesso em: 6 ago. 2014.

BRASIL. Ministério da Saúde. Saúde bucal. Brasília, DF, 2006. Cadernos de Atenção Básica n. 17. Disponível em: <http://189.28.128.10o/dab/ docs/publicacoes/cadernos_ab/abcad17.pdf>.

Acesso em: 9 ago. 2014.

CAVACA, A. G. et al. A saúde bucal na mídia impressa: análise das matérias jornalísticas nos anos de 2004-2009. Ciência \& Saúde Coletiva, Rio de Janeiro, v. 17, n. 5, p. 1333-1345, 2012.

EMMERICH, A. A corporação odontológica e o seu imaginário. Vitória: EDUFES, 2000.

IBGE - INSTITUTO BRASILEIRO DE GEOGRAFIA E ESTATÍSTICA. Pesquisa de Orçamentos Familiares 2008-2009: Perfil de despesas no Brasil. Indicadores selecionados. Rio de Janeiro: IBGE, 2012.

HENRIQUEZ, V. E. P. Veiculação de informações sobre saúde bucal: 1980-1991. 1993. Dissertação (Mestrado em Odontologia) - Faculdade de Odontologia da Universidade Federal Fluminense, Niterói, 1993.

HERSCOVITZ, H. G. Análise de conteúdo em jornalismo. In: LAGO, C.; BENETTI, M. Metodologia de pesquisa em jornalismo. Petrópolis: Vozes, 2007. p. 123-142.

HOSSEINPOOR, A. R.; ITANI, L.; PETERSEN, P. E. Socio-economic inequality in oral healthcare coverage: results from the World Health Survey. Journal of Dental Research, Washington, DC, v. 91, n. 3, p. 275-281, 2012.

MATOS, D. L. et al. Projeto Bambuí: avaliação de serviços odontológicos privados, públicos e de sindicato. Revista de Saúde Pública, São Paulo, v. 2, n. 36, p. 237-243, 2002. 
MINAYO, M. C. S. O desafio do conhecimento: pesquisa qualitativa em saúde. 11. ed. São Paulo: Hucitec, 2008.

OLIVEIRA, V. C. A comunicação midiática e o Sistema Único de Saúde. Interface Comunicação, Saúde, Educação, Botucatu, v. 4, n. 7, p. 71-8o, 2000.

OLIVEIRA, V. C. Os sentidos da saúde nas mídias jornalísticas impressas. RECIIS - Revista Eletrônica de Informação e Inovação em Saúde, Rio de Janeiro, v. 6, n. 4, 2013. Suplemento. Disponível em: <http://www.reciis.icict.fiocruz.br/ index.php/reciis/article/view/622/1262>. Acesso em: 25 abr. 2014.

PERES, K. G. et al. Redução das desigualdades sociais na utilização de serviços odontológicos no Brasil entre 1998 e 2008. Revista de Saúde Pública, São Paulo, v. 46, n. 2, p. 250-258, 2012.

ROCHA, R. A. C. P.; GOES, P. S. A. Comparação do acesso aos serviços de Saúde Bucal em áreas cobertas e não cobertas pela Estratégia de Saúde da Família em Campina Grande, Paraíba, Brasil. Cadernos de Saúde Pública, Rio de Janeiro, v. 12, n. 24, p. 2871-2880, 2008.

RONCALLI, A. G. Projeto SB Brasil 2010: Pesquisa Nacional de Saúde Bucal revela importante redução da cárie dentária no país. Cadernos de Saúde Pública, Rio de Janeiro, v. 27, n. 1, p. 4-5, 2011.

RONZANI, T. M. et al. Mídia e drogas: análise documental da mídia escrita brasileira sobre o tema entre 1999 e 2003. Ciência \& Saúde Coletiva, Rio de Janeiro, n. 14, v. 5, p. 1751-1762, 2009.
SANTOS, S. B. Mídia e saúde: os editoriais sobre saúde do periódico pernambucano Jornal do Commercio. Reciis - Revista Eletrônica de Comunicação e Informação \& Inovação em Saúde, Rio de Janeiro, v. 6, n. 4, 2012. Disponível em: <http:// www.reciis.icict.fiocruz.br/index.php/reciis/article/ view/670/1285>. Acesso em: 25 abr. 2014.

SHOEMAKER, P. J.; REESE, S. D. Mediating the message, theories of influences on mass media content. 2. ed. White Plains: Longman, 1996.

THIEDE, M.; AKEWENGO, P.; MCINTYRE, D. Explorando as dimensões do acesso. In: MCINTYRE, D.; MOONEY, G. Aspectos econômicos da equidade em saúde. Rio de Janeiro: Editora Fiocruz, 2014. p. 137-161.

THIEDE, M.; MCINTYRE, D. Informação, comunicação e acesso equitativo aos cuidados de saúde: um comentário conceitual. Cadernos de Saúde Pública, Rio de Janeiro, v. 24, n. 5, p. 11681173, 2008.

TRAVASSOS, C.; CASTRO, M. S. M. Determinantes e desigualdades sociais no acesso e na utilização de serviços de saúde. In: GIOVANELLA, L. et al. Políticas e sistemas de saúde no Brasil. Rio de Janeiro: Fiocruz, 2012, p. 183-206.

WOLF, M. Teorias da comunicação. 5. ed. Lisboa: Editorial Presença, 1999.

\section{Contribuição dos autores}

Esposti e Cavaca participaram de todas as etapas gerais do estudo: concepção do estudo, planejamento, construção, análise e interpretação dos dados e redação do artigo. Côco participou da análise e interpretação dos dados e da redação do artigo. Santos-Neto e Oliveira participaram da interpretação dos dados e da revisão crítica do artigo.

Recebido: 15/10/2014 Reapresentado: 26/06/2015

Aprovado: 17/08/2015 